\title{
FROM GRADUATE TO UNDERGRADUATE
}

Translating a Successful Online Graduate Model for Undergraduate Teaching

Paul Darbyshire and Geoff Sandy

School of Information Systems, Victoria University, Australia

Paul.Darbyshire $a$ vu.edu.au Geoff.Sandy@ $(\bar{y} u . e d u . a u$

\begin{abstract}
Online teaching has not as yet penetrated too deeply into the undergraduate culture, and most successful programs are targeted towards vocational or postgraduate courses. It is often argued that postgraduate students are more likely to have the skills necessary to survive in an online environment. However, there is a large cross-section of undergraduates, which would find benefits in the flexibility of online delivery and will also possess the skills necessary to successfully complete online courses. Anecdotal evidence indicates a changing profile for undergraduate students where many continue to work part-time while studying. The work-experience gained by these students can give them experiences, which can be used to construct their learning experience in a synergistic manner by utilization of an appropriate online paradigm. This paper details the experiences of translating a successful online teaching approach used in a fully online postgraduate course to an undergraduate program.
\end{abstract}

Key words: Online learning, online model, undergraduate teaching, feedback loop.

\section{INTRODUCTION}

Despite initial opposition, online teaching is now becoming a component of many university courses, and in some cases is the only delivery paradigm implemented. Online teaching has not as yet penetrated too deeply into the undergraduate culture, and most successful programs are targeted towards vocational learning or postgraduate courses. Postgraduate students, particularly those who are working and trying to obtain their qualification at the same time, benefit from the flexibility that a fully online course can offer. Additionally, postgraduate students are more likely to have the skills necessary to survive in an online environment, where it can be argued that 
undergraduate students are obtaining these skills. However, not all undergraduate students are alike, and there is a large cross-section which would find benefits in the flexibility of online delivery and possess the skills necessary to successfully complete online courses.

This paper details the experiences of translating a successful online teaching paradigm used in a fully online postgraduate course to an undergraduate program. The online teaching model is based on a semiSocratic paradigm and is largely constructivist in nature. This model is successfully used in the largest international fully online Master of Computer Science course, and targets working professionals too busy to attend the traditional classroom environment. By implementing this model, the School of Information Systems at Victoria University successfully conducted an online pilot in for its Faculty core subject in semester 12004 for undergraduate students. The pilot deliberately targeted part-time students who were self-motivated and willing to share personal experiences to make best use of the paradigm. These students, while not possessing all the proficiency of a postgraduate student, generally possess the motivation and skills necessary to survive in the online environment.

In the following sections, a brief literature review is presented on teaching and learning paradigms and online approaches. This is followed by a description of the approach translated from a graduate program for use in the undergraduate subject. A description of the implementation of the approach is then given and the results from the pilot online subject are presented.

\section{LITERATURE REVIEW}

The dominant paradigm for teaching and learning at Universities has been described as Pedagogy (KIT 2003; Heuer and King 2004). An emerging paradigm suitable for online teaching and learning has been referred to as Andragogy (KIT 2003; Heuer and King 2004). The major differences between the paradigms are summarized in Table 1, Two Teaching and Learning Paradigms (KIT 2003; Pelz 2004). The emerging paradigm prefers the term facilitator or moderator to that of teacher or instructor. In an online environment the term is e-facilitator or e-moderator (Salmon 2000; Heuer and King 2004). 
Table 1. Two teaching and learning paradigms

\begin{tabular}{ll}
\hline \multicolumn{1}{c}{ Pedagogy } & \multicolumn{1}{c}{ Andragogy } \\
\hline $\begin{array}{l}\text { Learner is dependent on the teacher } \\
\text { Teacher initiated transmission of information }\end{array}$ & $\begin{array}{l}\text { Learner is self directing } \\
\text { Welcomes the learners experience as a } \\
\text { valuable contribution }\end{array}$ \\
$\begin{array}{l}\text { Learning is subject centred } \\
\text { Assumes if a student is not ready then no } \\
\text { learning will take place }\end{array}$ & $\begin{array}{l}\text { Learning is more life centred or task oriented } \\
\text { Students will be ready to perform more } \\
\text { presumes students are motivated by external }\end{array}$ \\
\hline
\end{tabular}

There are a number of approaches to the facilitation or moderation of an online class. Bedore et al. (1998) suggests a useful classification of Interactive, Bounded Interactive, Consultative, Independent and Special Configuration. The role of the facilitator varies with each approach principally because of the different levels of interactivity required. In the case of the Interactive approach where a class size is about 10-15 students and each student is expected to send 10-12 meaningful messages a week high level skills are required of the facilitator. However, for the Consultative approach where there are about 40-60 students the facilitators role is primarily used by the students as a subject matter expert. Such a model has greater similarity to some approaches belonging to the Pedagogy paradigm.

Despite initial opposition to online teaching and learning it is increasingly being adopted by Universities. Opposition usually criticizes the high demands and commitment together with the need for excellent timemanagement skills required by both facilitator and students (Haddad 2000; DiPaolo 1999). Again criticism is also made about the need for students to work independently and in isolation and with a lack of face-to-face personal interaction that results in impaired teaching and learning (Haddad 2000; DiPaolo 1999). Finally, criticism is made that students must be technologically literate and have access to the relevant technology (Haddad 2000; DiPaolo 1999).

In contrast the major benefits claimed for online teaching and learning stress the convenience and flexibility for the students and less interruption to careers from the learning (Davis 2001; FCIT 1999). Further it is claimed that online classes are richly diverse in terms of age and ethnicity and students are usually highly motivated (Davis 2001; FCIT1999). Proponents of online teaching and learning freely acknowledge that it is not suitable for everyone but will provide educational outcomes at least equal to similar offline environment (Dutton et al 2002; Neuhauser 2002). 
As mentioned previously this paper reports on the use of a hybrid online teaching and learning approach for undergraduate students in an Australian University, Victoria University. This approach is now described.

\section{AN ONLINE LEARNING APPROACH}

The online teaching and learning approach reported on here is based on a semi-Socratic ontology and is largely constructivist in epistemology. In terms of the approaches referred to previously it is a hybrid of the Interactive and Bounded Interactive. The approach is successfully used in the largest international fully online Master of Computer Science course of the University of Liverpool and its partner Laureate Online Education B.V. (formerly K.I.T. eLearning). It targets working professionals who find it difficult to attend the traditional classroom offline environment. These students are usually highly motivated, technologically literate and are willing to work independently and meet the high demand of time to be successful in the learning. They welcome the convenience and flexibility such approach gives them and view the interaction with a richly diversity classmates as a positive experience. The Masters course adopts best practice criteria as outline in MSACHE (2001)

The main features of the approach adopted here are described in Bedore et al (1998). The approach limits the class size to 15-20 students so as to achieve a high level of dialogue. However, the level of dialogue should be such that it does not impose an unreasonable load the students and is at a level that can be processed by the facilitator. As a rule of thumb if the message rate is in excess of 200 messages per workshop then this limit is reached. Messages represent a student's individual input including papers/ project work, assignments, responses to discussion questions as well as comments related to other students' input. Each student is expected to read all messages. This is a major reading and response load for students and for the facilitator who must manage this load while providing appropriate leadership and comments. This requires high-level facilitation skills.

Because of the strong partnership between Laureate and the University there is a strong commitment to the students' success. Each student is assigned a Program Manager who acts a personal contact throughout the course. The University exercises close quality control over the course content, quality of students and quality of facilitators. Faculty staff (facilitators) are supported by both Laureate and the University on both academic and technical dimensions. All facilitators must undertake and perform satisfactorily in an online training module before they can take a module. The first time a staff members takes a module the facilitator is 
mentored and evaluated. An unsatisfactory performance means no certification.

Students are admitted to the course after extensive consultation, verification of previous studies and a two-week orientation period where an online class is simulated. Students are clearly informed of assessment procedures as they are well documented and a through, transparent and fair grading process is used. Both student and staff are formally involved in the evaluation of each subject module given and a process is in place to evaluate the feedback and act

\section{IMPLEMENTATION OF THE APPROACH}

This successful approach was used by the authors from the School of Information Systems at Victoria University for a group of part-time students undertaking the core business subject titled Information Systems for Business. All students of the Bachelor of Business must take this core subject along with a number of other core subjects like Economics, Accounting and Management. This was a pilot study that deliberately targeted part-time students who were self motivated and willing to share work and life experiences to make a success of the opportunity to learn with this approach. However, the authors were conscious that there were differences between this group and those normally taking the Master of Computer Science. Specifically, they were younger and consequently had less work (and life) experience to draw upon together with the obvious differences and expectation associate with Undergraduate and postgraduate studies. The online learning software is WebCT Campus Edition, which is licensed to Victoria University and mandated for use by recipients of a Curriculum Innovation Grant (CIG). The authors are recipients of a CIG although they are also experienced in the delivery of PG courses using FirstClass software.

The existing undergraduate subject lectures were extensively rewritten to construct ten self-contained textual based seminars to relay the subject matter. Discussion questions were written for each of the seminars to initiate discussion on the seminar topics. Additionally, assignments were selected from the on-ground subject to complement the seminars. The length of the online pilot was reduced from the normal 12 week semester to 10 weeks, as online subjects are typically more intensive and of shorter duration. During the re-enrolment period, a self-evaluation survey was distributed to part-time students to enable them to determine if they were suitable for online learning. Finally, twenty students were selected from the respondents on a first come first serve basis, and the group was brought together for one 
meeting. During this meeting, all details of the conduct of the online class were explained, and an orientation was conducted on the use of the virtual class software.

\section{PILOT PROGRAM RESULTS}

The pilot online subject ran in semester 12004 , in a fully online asynchronous mode. The initial class size of 20 students stabilized to 14 students after the first three weeks. This represented a retention rate of $70 \%$, which is reasonable in a fully online class where the participants had not experienced the online paradigm previously. Only part-time students were actively recruited into the pilot online class, though the class did include two full-time undergraduate students. The class ran for 10 weeks, and a two week break was inserted after week 5 to break the online semester into manageable tasks. At the end of week 10, each student was sent a subject feedback form so data could be gathered concerning satisfaction with various components of the online subject.

The feedback consisted of 18 questions requiring a Likert scaled response of a number from one to five. A response of one indicates a poor or negative opinion of the point raised in the question, a response of five indicates a strong positive opinion of the point raised. The 18 Likert type questions are given in Table 2.

Table 2. Feedback Likert scaled questions

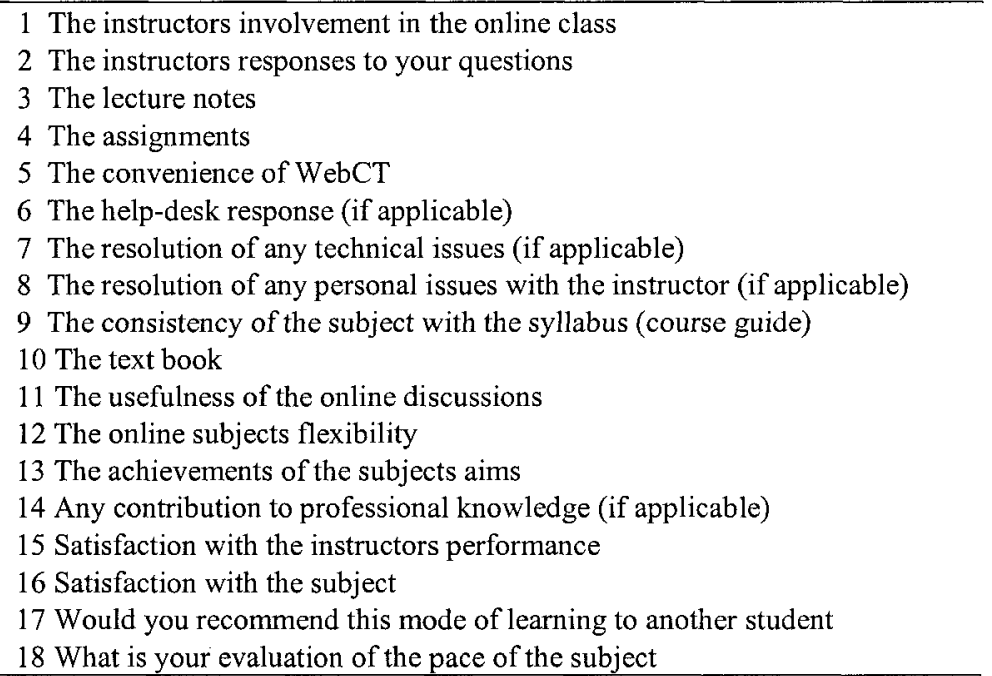


At the end of the feedback form, there are three open ended questions where the students are invited to comment further on other aspects which they were either happy of unhappy about. These questions are:

- Please describe in a few words one aspect of the subject you were most satisfied with.

- Please describe in a few words one aspect of the subject you were least satisfied with

- Is there anything else you would like to add regarding the online program?

At the end of the pilot in Semester 12004 , a total of five questionnaires were returned so no concrete conclusions can be drawn. However, the pilot experience is promising. The results from the Likert scaled questions of the questionnaire are shown in Table 3 along with comparative data from 4 classes of a graduate subject. The graduate subject is taught via Laureate through the University of Liverpool as part of the MSc in Computer Science. The figures from Laureate were collected from the subject feedback throughout 2002. The shaded column represents the averages for the Likertstyle questions for the undergraduate subject piloted at Victoria University. The same questionnaire was given to the undergraduate and Masters students, except the undergraduate questionnaire had two extra questions.

Table 3. Questionnaire Likert scaled responses

\begin{tabular}{ccccccc}
\hline Questions & Pilot Class & \multicolumn{5}{c}{ Comparative Graduate data (4 classes) } \\
averages & averages & class 1 & class 2 & class 3 & class 4 \\
\hline & & & & & & \\
1 & 4.4 & 3.9 & 4.1 & 3.7 & 3.5 & 4.4 \\
2 & 4.2 & 4.3 & 4.6 & 3.8 & 4.2 & 4.6 \\
3 & 4.2 & 4.5 & 4.6 & 4.1 & 4.5 & 4.8 \\
4 & 3.6 & 4.5 & 4.4 & 4.1 & 4.9 & 4.5 \\
5 & 5.0 & 4.0 & 3.9 & 4.0 & 3.8 & 4.1 \\
6 & 3.5 & 4.2 & 4.3 & 3.8 & 4.2 & 4.5 \\
7 & 4.0 & 4.0 & 4.1 & 4.0 & 4.0 & 4.0 \\
8 & 5.0 & 4.3 & 4.5 & 4.0 & 4.4 & 4.4 \\
9 & 4.4 & 4.2 & 4.3 & 4.1 & 4.3 & 3.9 \\
10 & 3.8 & 4.2 & 4.0 & 4.2 & 4.5 & 4.1 \\
11 & 5.0 & 4.0 & 4.2 & 3.8 & 4.1 & 4.0 \\
12 & 5.0 & 3.6 & 4.2 & 3.3 & 3.0 & 3.8 \\
13 & 4.6 & 4.2 & 4.5 & 3.9 & 4.1 & 4.4 \\
14 & 4.4 & 4.4 & 4.5 & 4.1 & 4.4 & 4.4 \\
15 & 4.4 & 4.2 & 4.7 & 3.6 & 4.0 & 4.6 \\
16 & 5.0 & 4.2 & 4.6 & 3.8 & 4.1 & 4.3 \\
17 & 4.6 & & & & & \\
18 & 4.0 & & & & & \\
\hline
\end{tabular}


A brief analysis of the data displayed in Table 3 can quickly highlight some of the differences between the graduate and undergraduate experience.

\subsection{Undergraduate Experience}

The undergraduate students responded more negatively to questions four and six, in the questionnaire, representing questions regarding the class assignments and the WebCT help-desk system. As the online class received assignments from the same material as the equivalent on-ground students, the response may indicate a lack of confidence in performing assignments by remote instruction. However, unless the on-ground students are similarly polled, it is difficult to speculate. Additionally, as there is not yet an online teaching culture established at Victoria University, the help-desk aspect of the online experience is not mature, and a poor result would have been expected.

There were a number of questions where the undergraduate response represented a more positive experience than the postgraduate one. These included: the instructors involvement in the class, the convenience of WebCT, the instructors help, the usefulness of the discussions, the flexibility of the subject, and the overall satisfaction of the subject. Of these points, question 12, 'the flexibility of the subject' stood out as the most significant positive difference between the undergraduate and graduate feedback. This is surprising given the similarity in the demographics of the students from both programs. The MSc program conducted by Laureate and the University of Liverpool is specifically targeted to working professionals; while in the pilot undergraduate subject at Victoria University, we intentionally targeted part-time students. The part-time students are normally in paid full-time employment and thus represent a group of students with similar demands on their time as the graduate students. However, as this pilot represents the first fully online class the students have participated in throughout their degree, the flexibility inherent in the subject design would have a great impact on their experience. The corresponding postgraduate subject would normally have been studied after a number of other online subjects, and thus at this stage, the postgraduate students would be more experienced and discerning of online class conduct.

As the undergraduate subject is designed around the online discussions, it is satisfying to see the relevant question ranking high in the student's perception. Above all else, it is the discussions of the paradigm we have adopted that drive the learning process. Thus satisfaction with the usefulness of the discussions would also directly relate to the overall satisfaction with the subject. This question also ranked very highly. Again, the lack of experience in online classes would most likely artificially inflate these 
figures until more experienced was gained. One of the more traditional methods of measuring the outcomes of the subject is of course the success rate of the students and the frequencies of the various grades received. This data is shown in Table 4.

Table 4. Final grade frequencies

\begin{tabular}{cccccc}
\hline $\mathrm{N} 2(0-39)$ & $\mathrm{N} 1(40-49)$ & $\mathrm{P}(50-59)$ & $\mathrm{C}(60-69)$ & $\mathrm{D}(70-79)$ & $\mathrm{HD}(80+)$ \\
\hline 0 & 0 & 3 & 6 & 5 & 0 \\
\hline
\end{tabular}

At first glance, the grades received seemed skewed towards the high end, but this reflects a common experience in online learning. Generally, online learning, while far more flexible, is also far more demanding. When the class numbers settle down after the initial drop-outs have taken place, those left are usually in for the long-haul, and represent those students more suited to the online paradigm. Such students tend to be self-motivated and hard working and will do well in the online or on-ground environment. This is usually reflected in the grades. It is interesting to note that although we intentionally targeted part-time students in the pilot online subject, we did have two full-time students participating. Two of the three pass (P) grades were allocated to those students. While we cannot draw conclusions based on two students, the follow-up class online class in Semester 2 is composed mainly of full-time undergraduates. It will be interesting to compare the performance levels between the two classes.

Although the grades shown in Table 4 represent an overall good performance, this was also one of the points of contention raised a number of times. At the end of the questionnaire, students were asked to comment on the thing they were least satisfied with in the subject. A number of them raised the question of the marking scheme...

I was going to moan about the marking system, but I see that's already been done, so I won't bore you any more ... actually, I will ... it was a bit of a pain when everybody handed in their DQ's and stuff on the last night of the week...

... the grading scheme seemed a bit harsh. You had to work very hard every week to had a hope of getting a HD, and the marking scheme made this very difficult...

In retrospect, we adopted the same grading scheme as the one used in the online MSc. This represented a requirement for a level of work that overly harsh in order to receive a high grade in an undergraduate first year subject. The grading scheme has been reviewed and adjusted for future classes and we will shortly have results from the next class due to finish in Semester 2 2004. 


\subsection{Instructor's Experience}

The experience of an instructor is always subjective, but from an instructors' perspective, the online class performed well in comparison to the experience of the graduate class. There were a number of notable differences, but these were mainly at the beginning of the semester when the students were becoming familiar with the online paradigm. More motivation in terms of instructor postings was required to initiate discussions early in the semester. There was more reluctance to discuss personal experience; however as the semester progressed, the students became more responsive to the questions. Towards the end of the semester, the discussion frequencies and quality of the postings reached very satisfying levels.

One of the concepts that seemed more difficult for the undergraduate class to grasp was that of synergy generated from the postings. The paradigm works well when student discuss regularly over a period of days. In fact, while this was a requirement, it was difficult to impress on many that it was imperative for the paradigm to work effectively. There was a particular cohort that seemed to post everything on the last night of the week, and technically, while meeting the required number of postings, didn't add to the synergy of the group during the week. Unfortunately, the only thing to change the behaviour pattern was the allocated marked for the week.

\section{CONCLUSIONS}

Many of the successful online programs are directed towards the graduate market. It is argued that at the graduate level, students have the commitment and the skills necessary to survive in the online environment. An online subject can be as, or more, demanding than the equivalent on-ground subject. However, the flexibility inherent in online approaches is attractive to people with busy life styles and demands made by family and career. There has not been a deep penetration of online learning into undergraduate culture, and it is uncertain if successful approaches used in graduate programs will be successful in undergraduate programs.

This paper has presented the results of translating a successful online approach used in a fully online MSc to an undergraduate subject. The subject was specifically marketed to part-time undergraduate students. This represented a section of the student population that would have a similar demographic to those undertaking the MSc, but with far less academic background. The outcomes for the students undertaking the online subject were promising, despite criticism of the marking scheme that was retained from the MSc program. The feedback from the end-of-subject questionnaires 
overall showed a more positive response to many of the aspects of the online subject, particularly the flexibility and overall satisfaction. However, given the number of returns and the first-time experience for the students, it may be difficult to draw concrete conclusions.

Given the positive outcomes for the students and the flexibility offered to an often-neglected sector of the student population, the pilot subject seemed very successful. However, to judge effectively the success of this particular paradigm for undergraduate teaching, classes need to be conducted with a more representative cross section of the student population. Currently, such a class, with full-time students making up the majority is due for completion in semester 2, 2004.

\section{REFERENCES}

Bedore G, Bedore M and Bedore G (1998) Online Education: The Future is Now, Art Press. Davis A (2001) Conversion from Traditional Distance Education to Online Courses, Programs and Services. International Review of Resaerch in Open and Distance Learning, January.

DiPaolo A (1999) Online Education: Myth or Reality? The Stanford Online Experience, Proceedings on Online Education, Berlin.

Dutton J, Dutton M and Perry J (2002) How do Online Students differ from Lecture Students. JALN, Vol. 6, No. 1.

Florida Center for Instructional Technology (FCIT) (1999) Benefits of Distance Learning. A Teachers Guide to distance Learning, Chapter 3.

KIT (2003). Seminar Week 2, Online Training.

Knowles M ( unknown) Andragogy. http://www.dmu.ac.uk/ jamesa/learning/knowlesa.htm

Date accessed 18 Octber 2004.

Haddad W (2000) Higher Education:The Ivory Tower and the Satellite Dish, TechKnow Logia, Vol. 2, No. 1.

Heuer B and King K (2004) Leading the Band: The Role of the Instructor in Online Learning for Educators, Journal of Online Education,

Holocomb L, King F and Brown S (2004) Student Traits and Attributes Contributing to success in Online Courses: Evaluation of University Online Courses. Journal of Interactive Online Learning, Vol. 2, No. 3.

MSACHE (2001) Best Practices for Electronically Offered Degree and Certificate Programs. Commission on Higher education, Middle States Association of Colleges and Schools.

Neuhauser C (2002) A Comparative Study of the Effectiveness of Online and Face-to-Face Instruction. The American Journal of Distance Education, Vol 16, No 2.

Pelz B (2004) (My) Three Principles of Effective Online Pedagogy, JALN, Volume 8, Issue 3, pp. 33-46.

Rivera J and Rice M (2002) A Comparison of Student Outcomes and Satisfaction between Traditional and Web Based course Offerings. Journal of Distance Education Learning Administration, Vol.5, No. 3.

Salmon G (2000) E-Moderating: The Key to Teaching and Learning Online, Kogan Page. 